\title{
Study of the influence on emotional work of contract nurses on Job Burnout
}

\author{
Changping Song, Weiwei Fan, Chenxu Zhao and Ling Gao ${ }^{a}$ \\ Beihua University, Jilin City, Jilin Province, 132021 China
}

\begin{abstract}
Discuss the influences of contract nurse' emotion work on job burnout and provide theoretical basis for reducing the job burnout of perception level which caused by the emotion work of contract nurse. Methods: The data which comes from the questionnaire survey about the Chinese edition of Maslach job burnout scale for 298 contract nurse is used for statistical analysis by SPSS11.5 software. Results: The current contract nurse is more inclined to "surface acting" on working and the nurses have a lower level on "depersonalization" $(\mathrm{P}<0.05$, the difference is statistically significant) and the more "deep acting" are used, the lower level of job burnout. There is a various factors' correlation between contract nurse's emotion work and job burnout. Conclusions: The "surface acting" could increase job burnout, "deep acting" and emotion expression requirements could increase personal job satisfaction.
\end{abstract}

\section{Introduction}

Emotional work refers to the individual who produces a kind of facial or body expressions which can be observed by public that made by the emotional management, including the surface acting, deep acting and emotional expression requirements. Job burnout is individual who cannot successfully deal with the working pressure after the extremely reaction and the individual is under pressure for a long time experience with the emotion, attitude and behavior of state failure, including emotional exhaustion, depersonalization and diminished personal accomplishment[1]. The development of market economy and the increase of social competition make our country is entering the peak of job burnout, more happened in the service staff, among which the medical staff in the second place. Contract system nurses, namely, the employment of nurses referring to the hospital are not a member of the official establishment, but the hospital signed a labor contract with the labor contract nurses. There is a gap between the contract nurses and formal preparation nurses in wages, insurance, promotion and so on. resulting in Contract nurses' job satisfaction levels drop, turnover rate increase, Seriously affect the stability of the nursing team, also bring adverse effect to the quality of care. This project investigates and analysis about the effect of contract nurses' emotional work on job burnout, in order to provide theoretical basis for reducing the perceived level of occupational burnout caused by the emotional work of contract nurses.

\footnotetext{
${ }^{a}$ Corresponding author : 364760038@qq.com

This subject is the social development project of Jilin science and Technology Bureau, Jilin province.
} 


\section{Research objects}

In June 2014, a questionnaire survey was used to investigate the contract system of 298 nurses from different departments in the Affiliated Hospital of Beihua University. were female, age 22-37 years old average 29 years old, department of internal medicine nurses 158 , accounting for $53.2 \%$, surgical nurses 140 , accounting for $46.8 \%$.

\section{Method of investigation}

First of all, trained the project team, to grasp the Emotional Workload Scale, the assessment method of nurses' Job Burnout Scale Questionnaire and the main content. The nurses' Emotional Workload Scale and nurses' Job Burnout Questionnaire were distributed to the 298 contract nurses who were investigated. The nurses' Emotional Workload Scale and nurses' Job Burnout Questionnaire were distributed by researchers to the 298 contract nurses who were investigated, and explained the purpose, details and filling method of the survey with consent of the person being investigated. The survey subjects completed the questionnaire in an anonymous manner, were collected questionnaire right away to ensure accuracy. Last Statistical analysis the obtained data by SPSS11.5 software package

\section{Investigation tool}

Questionnaire is the Chinese version of Maslach Burnout Inventing[2]. Referring to Ye Zhihong[3] and other domestic norm of the diagnosis standard of occupational burnout emotional exhaustion is greater than or equal to 27,depersonalization is greater than or equal to 8,personal accomplishment is less than or equal to 24 as the critical value. Li YongXin[4] divides burnout degree into four types:(1) zero burnout: in the assessment of occupational burnout, three factor scores were lower than the critical value; (2) mild burnout: in the assessment of burnout, a factor score higher than the critical value; (3) moderate burnout: in the assessment of burnout, two factor scores were higher than the critical value; (4) high burnout: in the assessment of occupational burnout, three factor scores were higher than the critical value.

\section{Results and discussion}

\subsection{Nurses emotional work condition}

Table 1. Emotional work of contract nurses $(n=298)$

\begin{tabular}{lccc}
\hline Emotional labor scores & Maximum & Minimum & Average+/-Standard deviation \\
\hline Surface acting & 2.53 & 4.14 & $4.58 \pm 0.36$ \\
Deep acting & 1.47 & 3.48 & $3.09 \pm 0.44$ \\
Emotional expression & 1.67 & 4.78 & $3.76 \pm 0.34$ \\
requirements & & & \\
\hline
\end{tabular}

Table 1 shows that current contract nurses are more inclined to work with surface acting, especially when nurses were suffer from severe emotional events, or at a high degree of job burnout or excessive emotional state. They don't want to do the positive cognitive adjustment on the subjective, but adopt appropriate emotion by surface acting. There are also some contract nurses tend to a pleasant mood on surface because of the needs of nursing work, but no really treat patients from deep inside. 


\subsection{Comparison nurses' occupational burnout scores with Maslach(n=309)}

Table 2. Comparison Contract nurses' occupational burnout scores with Maslach

\begin{tabular}{lcccc}
\hline & The number of & $\begin{array}{c}\text { emotional } \\
\text { exhaustion }\end{array}$ & depersonalization & $\begin{array}{c}\text { Loss of personal } \\
\text { accomplishment }\end{array}$ \\
\hline $\begin{array}{l}\text { Maslach } \\
\text { experiencegroup }\end{array}$ & 309 & $21.19 \pm 9.53$ & $7.12 \pm 2.21$ & $36.54 \pm 7.34$ \\
control group & 149 & $29.53 \pm 11.02$ & $9.37 \pm 2.14$ & $39.02 \pm 7.98$ \\
& 149 & $28.98 \pm 11.84$ & $9.65 \pm 2.23$ & $39.12 \pm 8.06$ \\
P & & 0.041 & 0.018 & 0.023 \\
\hline
\end{tabular}

Table 2 shows that contract nurses performance moderate emotional exhaustion, personal work without a sense of accomplishment and low depersonalization. Compared with the Maslach, the depersonalization of the nurse group is seriously low. $(\mathrm{P}<0.05)$ Main performances are emotional fatigue, lack of sympathy for patients, be incompetent for the job, and lack of personal accomplishment, etc..

\subsection{The relationship between emotional work and job burnout of contract nurses}

Table 3. The relationship between emotional work and job burnout of contract nurses

\begin{tabular}{lccc}
\hline Project & $\begin{array}{c}\text { emotional } \\
\text { exhaustion }\end{array}$ & depersonalization & $\begin{array}{c}\text { Loss of personal } \\
\text { accomplishment }\end{array}$ \\
\hline Surface acting & 0.165 & 0.121 & 0.144 \\
$\begin{array}{l}\text { Deep acting } \\
\begin{array}{l}\text { Emotional expression } \\
\text { requirements }\end{array}\end{array}$ & 0.015 & 0.021 & 0.147 \\
\hline
\end{tabular}

Table 3 shows that there is a correlation between emotional work and job burnout of contract nurses. The more contract nurses use the deep acting, the lower gain the level of job burnout; The more contract nurses use the surface acting, the higher gain the level of job burnout.

\section{Conclusion}

The surface acting of emotion work has a significant predict effect on contract nurse's emotional exhaustion of job burnout and the expression of depersonalization. The more use of surface acting means the nurse in the work often camouflage the emotions and show excessive professional nursing behaviors. This expression behavior is inconsistent with cognitive evaluation and emotional experience will decrease self-sense of reality. The individual needs to pay more psychological resources which make it easier to fall into mental fatigue and to some extent is easier to burnout, aggravate emotional exhaustion and depersonalization. However deep acting pays more attention to inner experience, more adjustment from the perspective of cognitive evaluation to achieve the request of organizational emotional expression to individuals, which reflects that contact nurses coordinate individual inner feelings with their external emotions, the psychological processing will make people have more kinds of moods, it is helpful for nurse to manage emotions in the work better, alleviate burnout, and improve the nurse's personal achievement. Therefore, the "surface acting" could increase job burnout, "deep acting" and emotion expression requirements could increase personal job satisfaction.

\section{References}

1. Elloy DF, Terpening W, KohlsJ. Aeausal model of burnout Among selfmanaged-work teammembers[J].The Journal of Psychology,2001,135(3):321-334.

2. Feng Ying, Luo Hong, Ye Zhihong,ect. Job Burnout of Nurses[J].Chinese Mental Health Journal, 
2004,18(7):477-479.

3. Ye Zhihong, Luo Hong, Jiang Anli. Diagnostic standard and norms of Maslach Burnout Inventory for nurses In Hongzhou[J]. Chinese Journal of Nursing, 2008,43(3):207-208.

4. Li Yongxin, A comparative study of job burnout among two groups: based on the perspective of integration [D].Doctoral Dissertation of East China Normal University,2005:74-76. 